\title{
Study On the Detection and Warning System of Rice Disease Based on the GIS and IOT in Jilin Province
}

\author{
Guogang Zhao ${ }^{1,2, \mathrm{a}}$, Haiye $\mathrm{Yu}^{1,2, \mathrm{~b}}$, Guowei Wang ${ }^{1,2,3, \mathrm{c}}$, Yuanyuan Sui ${ }^{1,2, \mathrm{~d}}$, Lei \\ Zhang ${ }^{1,2, \mathrm{e}}$ \\ ${ }^{1}$ College of Biological and Agricultural Engineering, Jilin University,Changchun 130022,China; \\ ${ }^{2}$ Key Laboratory of Bionic Engineering,Ministry of Education,Changchun 130022,China; \\ ${ }^{3}$ School of Information Technology, Jilin Agricultural University, Changchun 130118,China \\ azhaoguogang2000@qq.com, bhaiye@jlu.edu.cn, ${ }^{c} 41422306 @ q q . c o m,{ }^{d}$ suiyuan0115@126.com, \\ ${ }_{\text {e_lei@jlu.edu.cn }}$
}

\begin{abstract}
Rice disease which has intimate connection with climate has a tremendous impact on the output and quality of rice. The present paper intends to construct a web to monitor the climate environment of rice growing by making use of IOT(Internet of things) and to exactly spot the regions where related statistics are collected and warned by taking advantage of GIS and then by means of C\# language and SQL server, to realize the visuality of system and database management. Thereafter, under fuzzy clustering algorithm based on the collected environmental data to judge whether the rice disease will happen and need warning in case that unnecessary lose is caused.The finding reveals that by practicing the present system in Rice Disease Department of Academy of Agricultural Science of Chang Chun, the system functions well in detecting the serious disease.
\end{abstract}

Keywords: GIS; Internet Of Things; rice disease; monitor; warning

\section{Introduction}

Jilin Province is advantageous in grain production and is also one of important commercial commodity grain base. Rice is one of three kinds of corps in jilin province and thus rice disease has influenced the output and quality of rice. Hense, to construct the disease detection system is necessary. Currently, the previous studies on rice disease are as follows: Su Mingyong with his fellows collected the monitoring data and analyzed the data in order to spread the information and put forward the scientific motivation for treatment[1,2]. Yuan Tao with his co-workers realized the prior warning by means of case inference technology based on the symptoms of disease[3]. However, the above studies obviously just focused on the disease rather than preventing before the disease. Xiao Deqin studied wireless water conductor collecting the data to analyze the water in the rice field, which innovate the previous studies lying in the wireless water conductor that is able to realize to conduct water on a wider range and more remote distance. Besides[4], there are more researches which are related to rice disease based on specialist system and database[5-9]. However, the 
approaches above are limited on the range of database to infer rather than realize the real-time monitoring based on the latest statistics and then make the policy.

The paper will build a wireless conductor net based on IOT mainly being reflected by water temperature, air moisture and daylight illumination of rice field to collect data on which the calculating ways are ensured and then days among monitoring which are mot the same as the set temperature humidity level and illumination are recorded to forecast whether there will be rice disease in the chosen rice field. At this time, GIS will work to present the current number for the above items and their corresponding location. If there needs warning, then the mark will be made in the map. Meanwhile, the message will be sent to the farmer by mobile phone in order to make exact measurement to save rather than lose. So the significance of the present study lies in the above innovation.

\section{System Design}

The $\mathrm{c} / \mathrm{s}$ construction model is adopted to realize the serving way of multiply customer enjoying the data. The framework of the system is as follows: (see fig. 1)

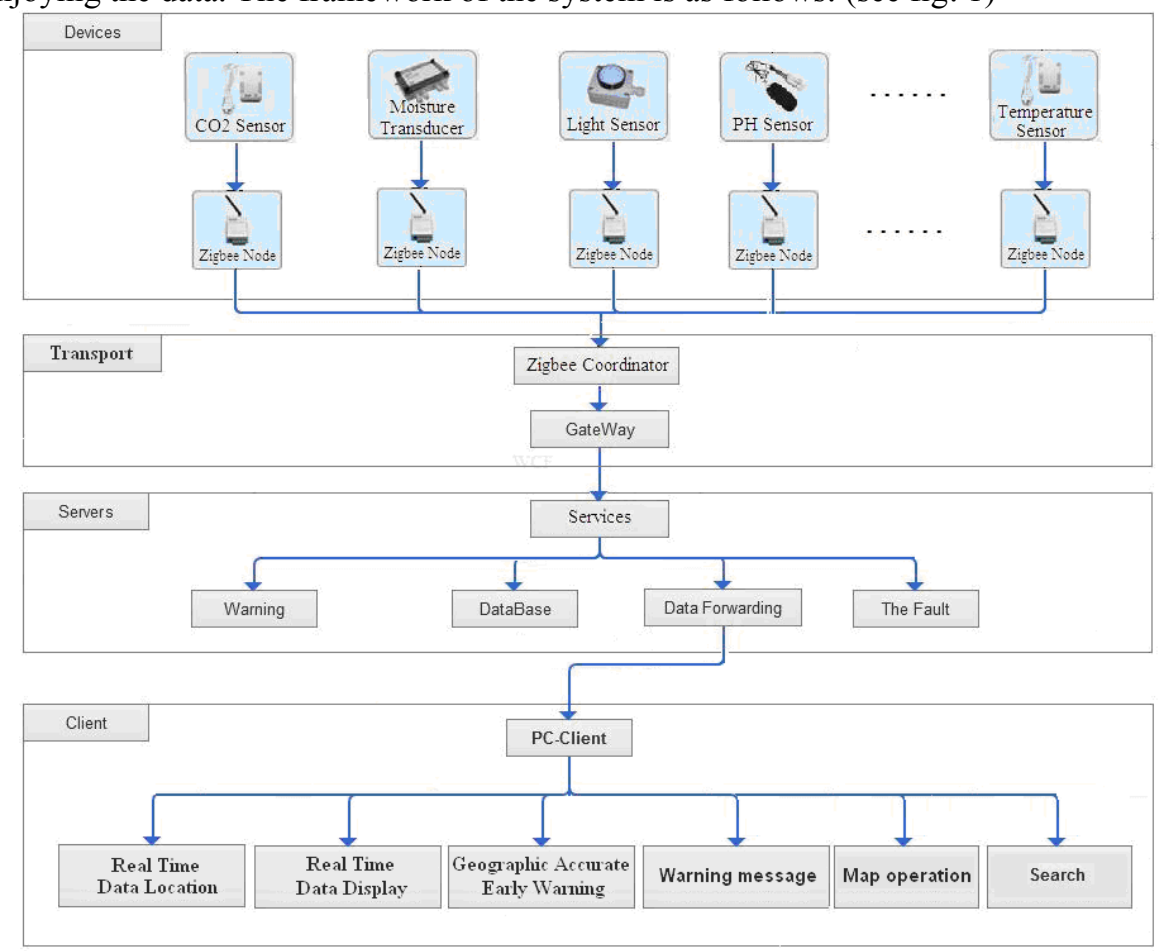

Fig. 1. the whole framework of system 


\subsection{Data Communication Agreement}

Data communication agreement is a series of agreement which aims to guarantee the communicators to effectively and reliably communicate within the data communication web. In the IOT, communication between server and hardware, between sever and various clients needs to be realized. Therefore, the present communication agreement is the key to ensure the function of system, which adopts statistics agreement as the follows: "data-node number-electricity-air-temperatureair humidity-illumination-water temperature." When the system receives the message from the monitoring equipment, the clipping is made according to the communication format to store in the right database.

\subsection{Data collection}

Data is collected by Socket programmer within C\# language. The working procedure is as fig.2.

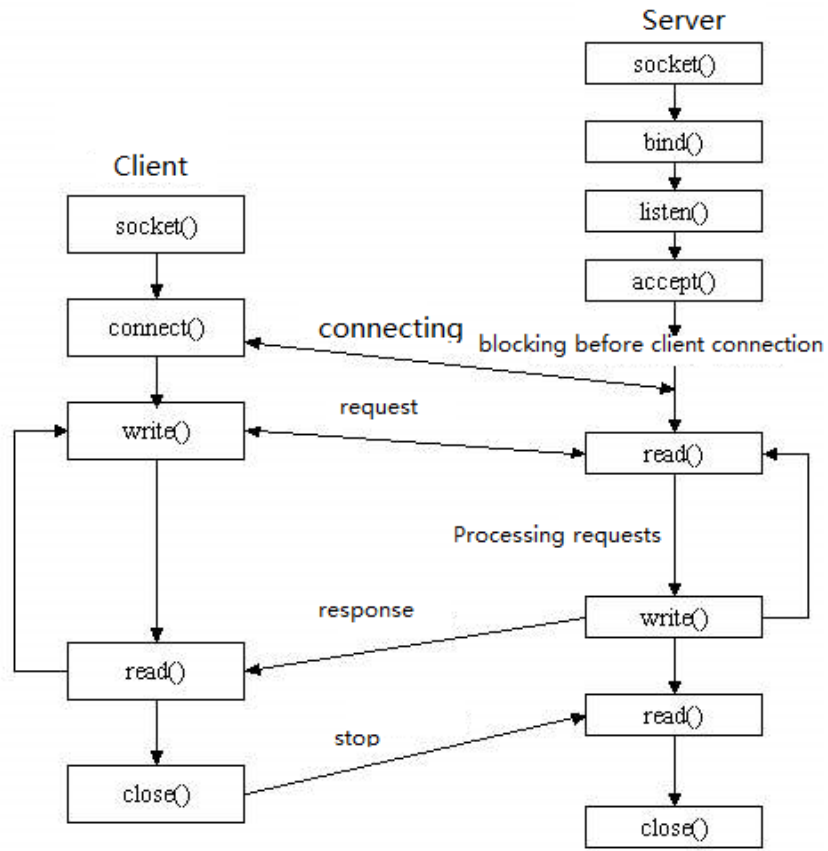

Fig. 2. Working procedure of Socket

\subsection{Function of GIS}

GIS not only presents where the data is from but also exactly tells the disease regions when the warning message is got, which facilitates the rice planter to prevent the disease beforehand. The GIS in present system takes advantage of MapObjects 
composition of ESRI Company in America. It consists of 46 parts, which constitutes 5 categories, individually being items of data operation (10), targets of map emergence( 17), objects of projection(8), objects of graphs(7) and items of address match (4). Among this kinds of C\# system, when any item is selected, the corresponding function can be made.

\subsection{Precautionary Model}

The model is realized by fuzzy clustering algorithm based on the data digging. The working procedure is as follows:

(1)The standardization of data weighing

To construct a primitive database D owing to the issues, among which $x_{i j}$ means the attributive value of $\mathrm{j}$ in the $\mathrm{i}$ sequence.

$$
D=\left[\begin{array}{cccc}
x_{11} & x_{12} & \cdots & x_{1 m} \\
x_{21} & x_{22} & \cdots & x_{2 m} \\
\cdots & \cdots & \cdots & \cdots \\
x_{n 1} & x_{n 2} & \cdots & x_{n m}
\end{array}\right]
$$

However, in reality, different data will have various weighing standard. So in order to compare numbers between different weighing standardization, data should be standardized between the sections $[0,1]$.

$$
x_{i j}^{\prime}=\frac{x_{i j}-\min \left\{x_{i j}\right\}}{\max \left\{x i_{j}\right\}-\min \left\{x_{i j}\right\}}
$$

(2) Construction of similarly fuzzy matrix

To calculate approach degree of fuzzy collection $i$ and $j$, the ways are amounts accumulation, coefficient of association, maximum and minimum method, arithmetic minimum mean method and algebra minimum mean method, absolute value pointing method, absolute value minus method, included angle cosine method. The present paper adopts the last one. The format is as follows:

$$
r_{i j}=\frac{\sum_{k=1}^{m} x_{i k} \cdot x_{j k}}{\sqrt{\sum_{k=1}^{m} x_{i k}^{2}} \cdot \sqrt{\sum_{k=1}^{m} x_{j k}^{2}}}
$$

Based on the formula in the above, the fuzzy matrix $R=\left(r_{i j}\right)_{n \times n}$ is got.

(3) Flowing cluster procedure

Fuzzy cluster analysis needs a equal fuzzy matrix which should be self-contradictory, symmetry and transitivity. Common fuzzy matrix has got the first two qualities. Thus 
we can make use of self-square blending to revise matrix $\mathrm{R}$ as matrix $t(R)$, that is,

$$
\begin{aligned}
& R \rightarrow R^{2} \rightarrow R^{4} \rightarrow \cdots \rightarrow R^{2(k+1)}=R^{2 k} \\
& R^{2}=R \circ R=\left(r_{i j}^{\prime}\right)_{m \times m}, \\
& r_{i j}^{\prime}=\max _{k=1}^{m}\left(\min \left(r_{i k}, r_{j k}\right)\right) \\
& t(R)=R^{2 k}=\left(t_{i j}\right)_{m \times m}
\end{aligned}
$$

After the $t(R)$ matrix has been got, the different number elements will be listed from the maximum to the minimum. $\lambda$ chooses the data according to the sequence to form the mobile cluster map which can be used to construct various models when different warning information is spotted.

\section{System Realization}

\subsection{Data Collection in real environment}

GIS and Socket functional programming will be made by use of C\# language and according to the definition of communication protocols, data capture will be received and obtain data collection sites and corresponding environmental parameter information will be obtained. The collected information is displayed in the system with the result in the fig.3:

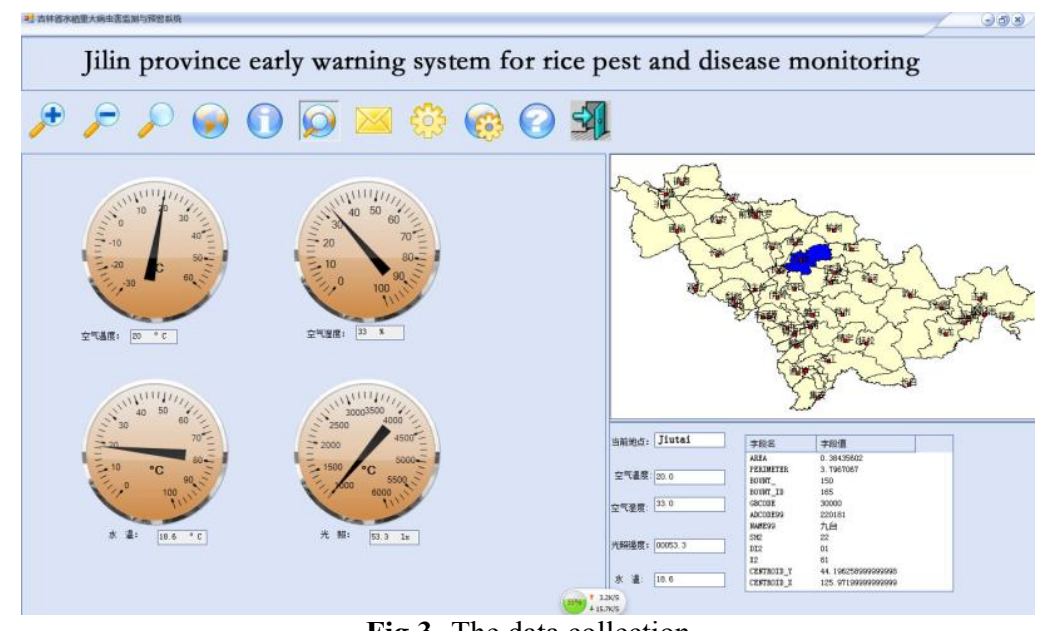

Fig.3. The data collection 


\subsection{Early warning of rice disease}

Through nearly twenty years of history data of Jilin Province are analyzed by fuzzy cluster analysis, according to the number of high temperature and high humidity weather, there are 4 categories: the first category includes no disease with high temperature and high humidity weather number less than 1 days; the second category involves $65 \%$ of rice having lighter diseases and 35\% medium disease condition; the third kind in high temperature and high humidity weather lasting $4 \sim 6$ days, $45 \%$ rice has mild disease, 36\% medium condition and 19\% heavy; fourth kinds of high temperature and high humidity weather more than 6 days, the rice disease occurred with $50 \%$ lighter, $23 \%$ moderate and $27 \%$ heavy.

When there is data acquisition, the system will judge according to the early warning model. If there is indeed warning, it will be marked on the map with red, at the same time, the early warning information in the form of text messages will be sent to mobile phone users; if there is no warning, no treatments. Fig. 4 shows the warning at the interface:

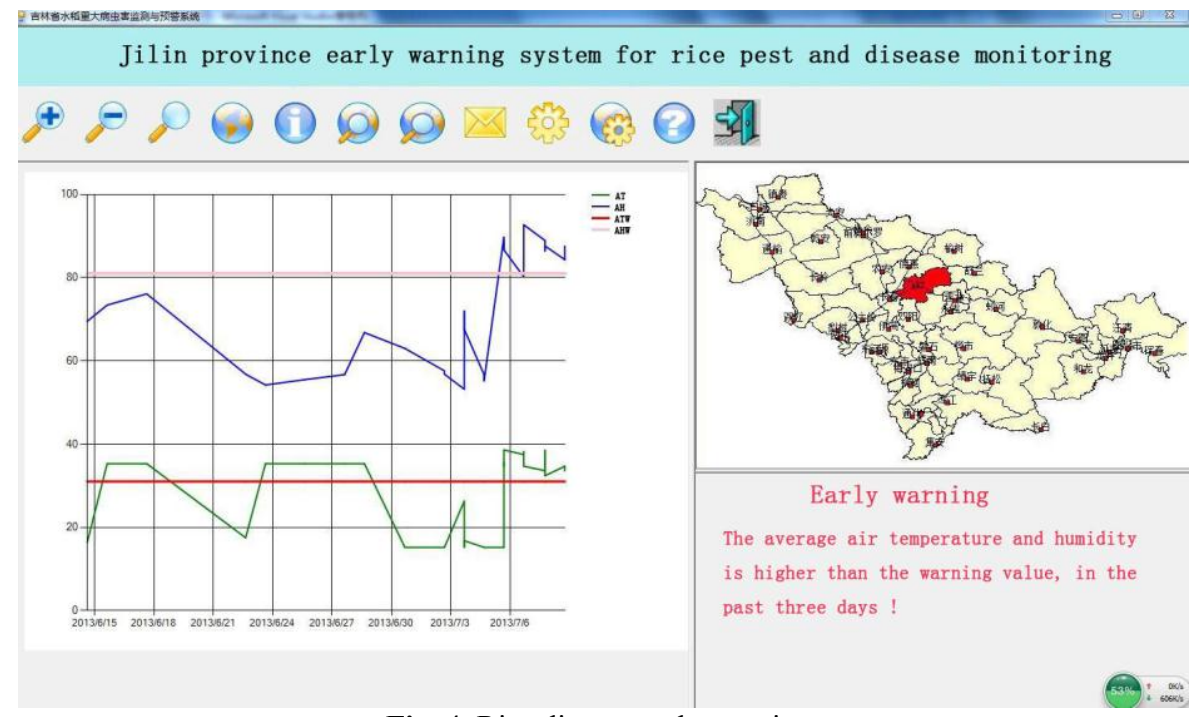

Fig. 4. Rice disease early warning

\section{Conclusions}

In this paper, by using GIS network and Socket programming technology with the use of $\mathrm{C \#}$ language, it realizes rice environmental data acquisition in Jilin Province, the display of geographic coordinates, the rice disease early warning and the geographical position of early warning and short message sending functions. Meanwhile, it presents a classification of occurrence of rice diseases so that the system in a timely manner to make judgments, suggesting that early warning information. 
The system was conducted with a year of operation in Changchun City Rice Research Institute, effectively to give advanced warning against rice blast effect and improve the quality and yield of rice, making project objectives.

\section{Acknowledgment}

Funds for this research was provided by National 863 subjects (2012AA10A506-4, 2013AA103005-04), Jilin province science and technology development projects(20110217), China Postdoctoral Science Foundation the 54th surface funded(2013M541308), Jilin University Young Teachers Innovation Project (450060491471).

\section{References}

1. Su MingYong,Liu Jitang,Construction of rice diseases and insect pests prevention and control system[J], Rural Economy and Science-Technology,, 2013,(9): 162-163.

2. Feng YINyin,Policy research of the rice pest control system in guangdong province[J]South China Rural Area, 2012, 28(7): 4-17.

3. Yuan Tao, Chen $\mathrm{Xu}$, Ma Chao, et al.Research on the Comprehensive Prevention and Control System of Rice Diseases and Insect Pests in Yangtze River Farm[J]Chinese Agricultural Science Bulletin, 2013,29(27):182-186.

4. Xiao Deqin, Gu Zhichun, Feng Jianzhao, et al. Design and experiment of wireless sensor networks for paddyfield moisture monitoring[J]. Transactions of the CSAE, 2011, 27(2): 174-179. (in Chinese with English abstract)

5. Chen $\mathrm{Xu}$,Wang Xiao,Yuan Tao,et al.Construction of information service platform of integrated pest control of rice based on service-oriented architecture(SOA)[J] Acta Agriculturae Shanghai ,2012,28(4):127-131.

6. Sun Min,Luo Weihong,Feng Wanli,et al.A Web-based expert system for diagnosis and control management of diseases in vegetable crops cultivated under protected conditions[J]Journal of Nanjing Agricultural University, 2014, 37(2): 7-14.

7. Lai JC, Ming B, Li SK, etal. An Image-Based diagnostic expert system for corn diseases [J] . Agricultural Sciences in China, 2010, 9(8):1221-1229

8. Gonzalez-Andujar JL. Expert system for pests, diseases and weeds identification in olive crops[J]. Expert Systems with Applications, 2009, 36(2):3278-3283

9. Abu-Naser SS. Kashkash KA, fayyad M. Developing an expert system for plant diseases diagnosis[J]. Journal of Artificial Intelligence, 2008, 1(2):78-85. 